\title{
Microstructured optical fiber sensor for soil moisture measurements.
}

\author{
A. Lopez Aldaba ${ }^{\mathrm{a}, \mathrm{b}, *}$, D. Lopez-Torres ${ }^{\mathrm{a}}$, M. A. Campo-Bescós ${ }^{\mathrm{c}}$, J. J. López ${ }^{\mathrm{b}, \mathrm{c}}$, D. Yerro ${ }^{\mathrm{c}}$, C. Elosua ${ }^{\mathrm{a}, \mathrm{b}}$, \\ F. J. Arreguia,b, J-L. Auguste ${ }^{\mathrm{d}}$, R. Jamier ${ }^{\mathrm{d}}$, P. Roy ${ }^{\mathrm{d}}$, and M. López-Amo ${ }^{\mathrm{a}, \mathrm{b}}$ \\ ${ }^{a}$ Universidad Pública de Navarra, Dept. of Electrical and Electronic Engineering, \\ ${ }^{b}$ Institute of Smart Cities (ISC), and IDISNA, Campus Arrosadia, 31006, Pamplona, Spain. \\ ${ }^{e}$ Electric and Electronic Engineering Department, Public University of Navarra, Edif. Los Tejos, Campus Arrosadía, 31006, Pamplona, Spain. \\ ${ }^{d}$ XLIM Photonics Department, UMR 7252, University of Limoges, CNRS, F-87000 Limoges, France \\ *aitor.lopez@unavarra.es; phone+3494816 9841; fax+34948 16 9720;
}

\begin{abstract}
A discrete sensor based on a $\mathrm{SnO}_{2}$-FP (Fabry-Pérot) cavity is presented and characterized in real soil conditions. Results are compared, for the first time to our knowledge, with a commercial capacitive sensor and gravimetric measurements improving some handicaps of capacitive commercial sensors and enabling precise local measurements.
\end{abstract}

Keywords: Soil moisture, Fiber Sensor, Humidity Sensing Photonic Crystal Fiber, Microstructured Optical Fiber.

\section{Introduction}

Soil moisture plays a key role in the hydrological cycle by controlling processes such as evapotranspiration or runoff and recharge of aquifers, just to mention some of them [1,2]. Therefore, soil water content data are very useful for the application and evaluation of hydrological models as well as for irrigation programming and optimization [2].

Among optical fibers, microstructured optical fibers (MOFs) have shown improved characteristics over conventional ones and a great potential for sensing applications. For instance, several pure silica suspendedcore fibers have been used in relative humidity $(\mathrm{RH})$ sensing [3].

FP cavities fabricated with MOFs are structures previously used for sensing like compact FP cavities for humidity between $40 \%-95 \%$ were reported [4].

Nanocoated based sensors have recently experienced a remarkable development [5]. Techniques in chemical deposition, such as sputtering, allow to control the morphology and thickness of the deposited coatings with high accuracy, and as a consequence, the final properties (sensitivity, kinetics) of the sensor.

In this work, an optical fiber sensor based on a sputtering deposition on a MOF is presented for real moisture measurements and compared simultaneously with a commercial capacitive sensor. The operation principle of the humidity optical sensor has been previously reported by authors [3] showing in detail the construction process of an optimized sensing head and its performance towards humidity variations. In the present paper, the authors propose, for the first time to our knowledge, a sensor that has been adapted and used for measurements in real soil conditions. Moreover, the authors also present a comparison between the performance of microstructured optical fiber sensors, a commercial capacitive sensor and gravimetric measurements; showing the pros and cons of each type of sensors (and remarking the advantages of fiber optics versus commercial capacitive ones).

\section{Experimental set-up and principle of operation}

\subsection{Principle of operation}

A Decagon 10HS capacitive sensor was used as a reference for the soil moisture measurements. Its operation principle consists of the measurement of the dielectric constant or permittivity of the soil to calculate its humidity content in a volume of $\sim 6.5 \mathrm{dm}^{3}$.

We have compared its measurements with an optical fiber Fabry-Pérot interferometer that was fabricated by splicing a single mode fiber (SMF) to a four-bridge MOF, whose end was cleaved, as shown 
in [3]. The MOF-FP optical fiber core was used as the substrate in a $\mathrm{SnO}_{2}$ coating-DC-Sputter deposition process also demonstrated in [3]. Due to the structure of the MOF used in this work, $\mathrm{SnO}_{2}$ can be deposited on the walls of the air holes of the short section of the MOF as well as on the top of the sensor head. $\mathrm{SnO}_{2}$ coating is highly sensitive to humidity changes the surrounding environment.

The interaction between $\mathrm{SnO}_{2}$ and $\mathrm{H}_{2} \mathrm{O}$ molecules is due to the phenomena called physisorption, by means of the adsorption/desorption of these molecules [6]. The molecules of water interact mainly with the surface of the deposited $\mathrm{SnO}_{2}$ thin film.

\subsection{Experimental set-up}

As it can be inferred, the sensing head is very fragile and moreover, dust inside the MOF holes or in contact with the $\mathrm{SnO}_{2}$ deposition could lead to measurement errors. In order to avoid this scenario, a protective cap was developed: it consists of a polyvinylchloride (PVC) tube with 4 arrays of drilled holes ( $1 \mathrm{~mm}$ of diameter) each $5 \mathrm{~mm}$ and a cap in one of its ends that enables the access of the optical fiber. Fig. 1 shows the real pictures of the protective cap and the final sensor installed inside the cap.

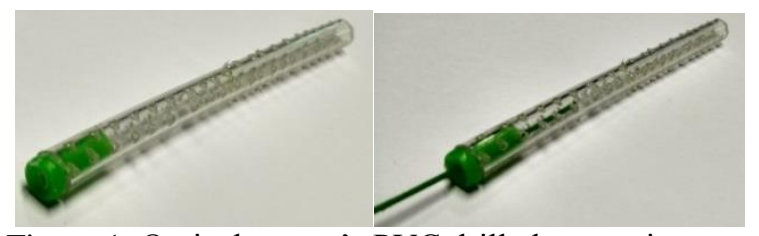

Figure 1. Optical sensor's PVC drilled protective cap.

The influence of the protective cap was verified before the installation in the soil to test the behavior of the sensing head.

A commercial interrogator equipment for FBGs based sensors (SM125, SMARTEC, Lugano, Switzerland) was used to illuminate the network and also to analyze the spectra of the signals reflected from the sensors. The interrogating equipment has a sampling frequency of $1 \mathrm{~Hz}$. The three sensors where buried in the soil.

A commercial $\mathrm{FBG}$ (sensitivity of $10 \mathrm{pm} /{ }^{\circ} \mathrm{C}$ ) was also monitored at the same time in order to measure the temperature variations inside the soil sample and compensate the optical sensor's deviations. Once known the variation of temperature through the FBG and the sensitivity of the MOF sensing head to temperature variations it can be directly compensated by subtracting the wavelength shift - FFT phase shift to the MOF's results.

In this study two different types of soil were tested in order to check the performance of the optical sensor in comparison with the capacitive one. The first porous media was a silica sand specified for use in pool filters, water purification and sports fields. This Soill texture was defined as sand (100\% sand). The second one (Soil2) was a mix of 50\% weight of the silica sand and 50\% weight of a contrasting natural soil texture classified as clay loam, $28 \%$ sand, $36 \%$ silt and $36 \%$ clay.

\section{Experimental results}

Long term measurements were performed in order to check the viability of the MOF-FP sensor. For this aim, the MOF-FP sensor was buried in the soil at $\sim 5 \mathrm{~cm}$ deep whereas the capacitive sensor was at $\sim 20 \mathrm{~cm}$. This difference must be underlined as it will affect directly on the results and it is produced due to the size difference of the sensors. On the one hand, MOF-FP sensor presents reduced dimensions allowing the moisture measurement within a small volume of soil while, on the other hand, capacitive sensors need a significant soil volume (as it will be described) to work properly. After the sensor's installation, water was carefully added to the soil until saturation (water dropping at the bottom of the sample - Relative humidity $=100 \%$ ). Every sensor was monitored each 10 minutes during $\sim 14$ days uninterruptedly showing the results in Fig. 2 . After $\sim 12$ days for Soil1 and $\sim 9$ days for Soil2, humidity was raised again until saturation to check the sensor's reversibility and adaptability to changing environments. 

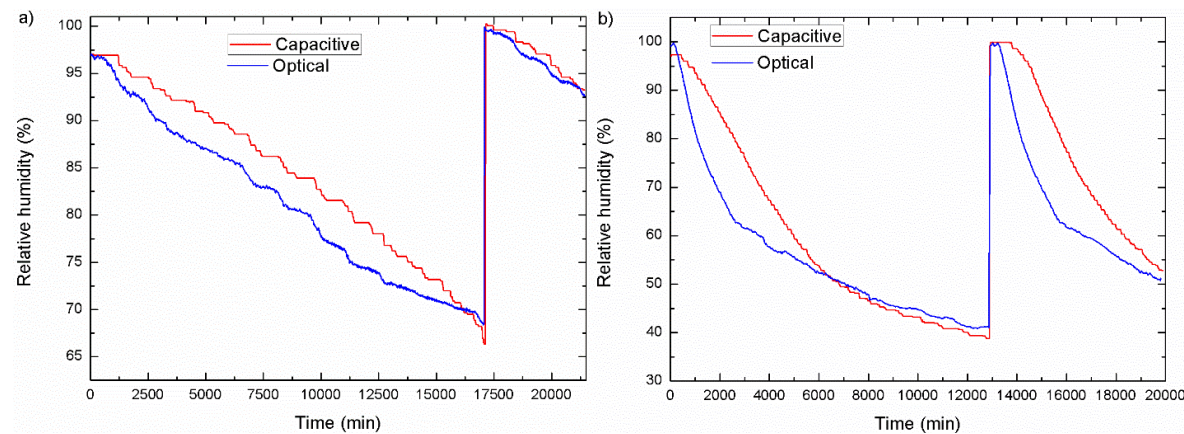

Figure 2. Capacitive and optical sensor's response towards soil humidity: (a) Soill and (b) Soil2.

There is a discrepancy in terms of kinetics of both sensors. This is due to the size of sensors and their sensing principles. On the one hand optical sensor measures the content of water directly in contact with the sensing head (changes in the refractive index of the surrounding media), performing point measurements and, on the other hand, capacitive sensor measures the content of water in a volume of $\sim 6.5 \mathrm{dm}^{3}$ of soil.

Another difference in the results can be seen in the $X$ axis of Figs. 2 (a) and (b). Fig. 2 (a) shows the results of the natural evaporation process in indoor conditions. As it can be seen, there is a very slow kinetic and humidity decreased $\sim 30 \%$ in 12 days. To accelerate this process, for Soil2 measurements (Fig. 2 (b)) two heating fans where placed close to the sample. Temperature variations were compensated as explained before. A significant difference can be seen as the humidity decreased $\sim 60 \%$ in 9 days.

\section{Conclusions}

In this work, a new sensing application of a microstructured optical relative humidity sensor has been presented for the first time. A comparison between a commercial capacitive FDR and the optical sensor has been carried out in two different types of soils during two weeks. Results show a good agreement between capacitive and optical measurements in the range 15\%-100\% humidity. Soil moisture can be measured with MOF-FP sensors with the advantages that they offer in comparison with traditional capacitive sensors: immunity to temperature variations (compensated), point measurements (against field measurements) and high multiplexing capability.

\section{ACKNOWLEDGEMENTS}

The Spanish Government projects TEC 2016-76021-C2-1-R, TEC2016-78047-R, TEC2016-79367-C2-2R, Innocampus and the Cost Action MP 1401, as well as to the AEI/FEDER Funds are acknowledged. Also, the authors thank to the Agricultural Engineering School for sharing the Hydraulic Laboratory.

\section{References}

[1] I. Rodríguez-Iturbe, Ecohydrology: a hydrologic perspective of climate-soil-vegetation dynamics, Water Resources Research, 36 (2000), 3-9.

[2] R. Nolz, G. Kammerer, P. Cepuder, Calibrating soil water potential sensors integrated into a Wireless monitoring network, Agricultural Water Management, 116 (2013), 12- 20.

[3] A. L. Aldaba, D. Lopez-Torres, C. Elosua, J. L. Auguste, R. Jamier, P. Roy, F.J. Arregui, M. LopezAmo, SnO 2-MOF-Fabry-Perot optical sensor for relative humidity measurements. Sensors and Actuators B: Chemical (2017).

[4] J. Mathew, Y. Semenova, G. Rajan, G. Farrell, Humidity sensor based on photonic crystal fibre interferometer, Electronics letters, 46(19) (2010), 1341-1343.

[5]F. J. Arregui, Sensors based on nanostructured materials, New York: Springer (2009).

[7] W. Schmid, Consumption measurements on $\mathrm{SnO} 2$ sensors in low and normal oxygen concentration (Doctoral dissertation, Universität Tübingen), (2004). 